\title{
Ethanol increases osteoclastogenesis associated with the increased expression of RANK, PU.1 and MITF in vitro and in vivo
}

\author{
NATSUMI IITSUKA, MAMIKO HIE, ATSUKO NAKANISHI and IKUYO TSUKAMOTO \\ Department of Food Science and Nutrition, Nara Women's University, Kita-uoya Nishi-machi, Nara 630-8506, Japan
}

Received January 24, 2012; Accepted March 5, 2012

DOI: 10.3892/ijmm.2012.974

\begin{abstract}
Ethanol has been known to induce osteopenia. However, the cellular and molecular mechanisms responsible for its effect have not been well characterized. This study investigated the effects of ethanol on bone metabolism and osteoclastogenesis using rats fed an ethanol-containing liquid diet (35\% of calories from ethanol) for 3 weeks. Ethanol increased the activities of bone tartrate-resistant acid phosphatase (TRAP) and cathepsin K, without affecting the levels of serum osteocalcin or bone alkaline phosphatase activity. Histological analysis showed an increased number of osteoclasts in the proximal tibia, but no significant change in the number of osteoblasts. The mRNA levels of receptor for activation of NF- $\mathrm{B}$ (RANK), c-fos, c-jun, TRAP and cathepsin $\mathrm{K}$ were significantly increased, although those of macrophage colony-stimulating factor and c-fms were unaltered. The mRNA and protein levels of PU.1 and microphthalmia-associated trascription factor (MITF) also increased. Further, the osteoclastic differentiation of bone marrow-derived macrophage/monocyte precursor cells (BMMs) in vitro was stimulated by ethanol. The increased osteoclastogenesis of BMMs was associated with increased levels of RANK, PU.1 and MITF expression, activated extracellular signal-regulated kinase (ERK), and reactive oxygen species (ROS). Higher lipid peroxide levels and lower glutathione levels were also observed in the serum of the ethanol-fed rats. These results suggested that ethanol promoted osteoclastogenesis by increasing RANK expression through increases in the production of ROS, activation of ERK and expression of PU.1 and MITF.
\end{abstract}

Correspondence to: Professor Ikuyo Tsukamoto, Department of Food Science and Nutrition, Nara Women's University, Kita-uoya Nishi-machi, Nara 630-8506, Japan

E-mail: itsuka@cc.nara-wu.ac.jp

Abbreviations: ALP, alkaline phosphatase; BMMs, bone marrowderived macrophage/monocyte precursor cells; CFU-M, colony-forming unit-macrophage; GSH, glutathione; RANK, receptor for activation of $\mathrm{NF}-\kappa \mathrm{B}$; RANKL, receptor for activation of NF- $\kappa \mathrm{B}$ ligand; ROS, reactive oxygen species; TBARS, thiobarbituric acid-reactive substances; TRAP, tartrate-resistant acid phosphatase

Key words: ethanol, osteoclastogenesis, receptor for activation of NF- $\kappa B$, PU.1, microphthalmia-associated trascription factor

\section{Introduction}

Alcohol-related damage to bone eventually results in osteopenia, a disease causing substantial morbidity and mortality (1). It is now well accepted that many disorders of bone, including osteopenia and osteoporosis, reflect an imbalance in the differentiation and function of two cell types, the osteoblast and osteoclast, which are responsible for bone formation and bone resorption, respectively $(2,3)$. This study aimed to clarify the effects of ethanol on the function and differentiation of osteoblasts and osteoclasts.

Ethanol dose-dependently reduces cell proliferation and alkaline phosphatase activity in osteoblasts. Moreover, the suppression of osteoblastogenesis is considered a major cause of ethanol-inhibited bone growth, bone loss and deficient bone repair (4). Ethanol has been also demonstrated to stimulate bone resorption (5) and osteoclastogenesis (6).

Osteoclasts, derived from bone marrow hematopoietic stem cells, are highly specialized multinuclear cells capable of resorbing bone (7). The number and activity of osteoclasts are determined by cell lineage allocation, the proliferation and differentiation of osteoclast precursors and the resorptive efficacy of mature osteoclasts (8). Osteoclastic differentiation requires macrophage colony-stimulating factor (M-CSF) and receptor for activation of NF- $\mathrm{kB}$ ligand (RANKL) (9). The binding of M-CSF to c-fms stimuates the expression of receptor for activation of NF- $\mathrm{kB}$ (RANK), the receptor of RANKL, in the hematopoietic osteoclast precursor cells. The binding of RANKL to RANK activates NF- $\kappa$ B and activator protein-1 (AP-1) and induces osteoclastic differentiation. Recently, we have reported that the suppression of RANK expression was associated with decreased levels of reactive oxygen species (ROS) $(10,11)$. Ethanol was reported to produce ROS in liver Kupffer cells and stellata cells (12), in the lung (13) and in osteoblasts (14). In osteoblasts, ethanol-generated ROS increased the expression of RANKL (14). We hypothesize that ethanol stimulates osteoclastogenesis by increasing RANK expression through direct action on osteoclast precursor cells in addition to the increased RANKL production in osteoblast/stromal cells.

In this study, we investigated the effects of ethanol on bone metabolism and on the gene expression involved in osteoclastogenesis in vivo and in vitro. Ethanol increased the activities and number of osteoclasts with a normal activity and number of osteoblasts. The increase in the number of osteoclasts was caused by the stimulation of osteoclastogenesis associated 
Table I. Primers used for quantitative real-time PCR.

\begin{tabular}{lll} 
Target & \multicolumn{1}{c}{ Forward primer sequence } & \multicolumn{1}{c}{ Reverse primer sequence } \\
\hline Actin & AGCCATGTACGTAGCCATCCA & TCTCCGGAGTCCATCACAATG \\
cathepsin K & TGTCTGAGAACTATGGCTGTGG & ATACGGGTAACGTCTTCAGAG \\
c-fos & TAGAGCCAGGTGCAACAGTG & CGCATAGGGTCTTCAAGCTC \\
c-jun & CTTCACCCTGCCTCTTCTCA & TCAAAGGGTTCAGCCTTCAG \\
M-CSF & TGAAGCAGAGCATGACCTTG & TAGTGGTGATGTGCCCATTG \\
MITF & CATCCAGGCAGAGACTGACA & TTCGCGCAGTGTAGATGAAC \\
OPG & TTGGAAGACATCCTGATGGAC & GCTGCTTGTTTCGAAGCTC \\
PU.1 & GAGTGTGCGAATGTGAGGAA & TGCTTTCGATGACGTCTCAC \\
RANK & TGGAGAAGCTGATGGCTTG & CCTTGTGCTTGGACGAGAA \\
RANKL & ATATGCCTGCATCCCCTGAA & TAGCCATCCGTTGAGTTGGA \\
TRAP & AGCGCAGATGGATCCTAACA & TCGAGTCCTGCAAACCTGTA \\
& CAGCCTTATTACCGTTTGC & GAATTGCCACACAGCATCAC
\end{tabular}

with the increased expression of RANK, PU.1 and microphthalmia-associated transcription factor (MITF) in osteoclast precursors and RANKL in osteoblasts/stromal cells.

\section{Materials and methods}

Animals and diets. Five-week-old male rats of the Wistar strain were purchased from Japan SLC, Inc. (Shizuoka, Japan) and housed individually in a temperature-controlled room with a 12-h light cycle. After a 1 week period of adaptation, the animals were randomly divided into 2 groups ( $n=8$ for each group); a control group and an ethanol-fed group. The ethanolfed rats were fed a Lieber-DeCarli ethanol-containing liquid diet with $35 \%$ of the calories derived from ethanol (F2LEW; Oriental Yeast Co., Ltd., Kyoto, Japan). The controls were pair-fed an alcohol-free isocaloric diet containing maltosedextrin (F2LCW; Oriental Yeast Co. Ltd., Kyoto, Japan). After 3 weeks, blood and femoral and tibial bone samples were collected under anaesthesia with sodium pentobarbital after overnight access to feed (non-fasting). Serum samples were used to determine the concentrations of ethanol, lipid peroxide, glutathione (GSH) and osteocalcin. After the removal of muscle and tendons, the tibial bone was used for a biochemical analysis, a histological analysis or preparing bone marrow cells. The bone marrow cells were used for the frequency analysis of the clonogenic precursors of osteoblasts and osteoclasts, and the in vitro colony formation assay. Animal experiments were performed in accordance with protocols approved by the Animal Care Research Committee of Nara Women's University.

Biochemical analysis. Serum concentrations of ethanol and osteocalcin were measured using a commercial ethanol diagnostic kit (Roche Diagnostics GmbH, Mannheim, Germany) and a Rat osteocalcin EIA kit (Biomedical Technologies, Inc., Stoughton, MA), respectively. Serum lipid peroxide levels were estimated as thiobarbituric acid-reactive substances (TBARS) calculated as malondialdehyde equivalents (15). Serum GSH levels were determined by the method of Ellman (16). The activities of ALP, TRAP and cathepsin K and the amounts of
Ca and hydroxyproline (Hyp) in the proximal tibia (the quarter from the aspect of the knee of the tibia) were determined as reported $(17,18)$.

Histomorphometry. The tibia was fixed in $4 \%$ paraformaldehyde, decalcified in $10 \%$ EDTA and embedded in paraffin. Sections ( $4 \mu \mathrm{m})$ were stained for TRAP activity using a leukocyte acid phosphatase kit (387A; Sigma) as described (17). Morphometric measurements of trabecular structure (trabecular bone volume, bone surface, thickness and number), and the number of osteoblasts (cuboidal cells on trabecular surfaces) and osteoclasts (TRAP-stained cells with more than 3 nuclei) were carried out at standardized sites $(300 \times 300 \mu \mathrm{m})$ under the growth plate in the metaphysis of the proximal tibia (19).

Analysis of the frequency of clonogenic precursors of osteoclasts or osteoblasts in bone marrow. The frequency of precursors of osteoclasts or osteoblasts in bone marrow was assessed based on a limiting dilution assay (20) as described previously (21). Briefly, the bone marrow cells were seeded into 96-well plates at 25, 50, 100 or 200 cells/well and cultured for 5 days in the culture medium [MEM containing $10 \%$ FCS, penicillin $(100 \mathrm{U} / \mathrm{ml})$, streptomycin $(100 \mu \mathrm{g} / \mathrm{ml}), 1 \%$ non-essential amino acid (NEAA) and $1 \%$ sodium pyruvate] with M-CSF (5 ng/ml) and RANKL ( $5 \mathrm{ng} / \mathrm{ml})$ for the assay of clonogenic osteoclast precursors, and at $3 \times 10^{5}, 6 \times 10^{5}, 10 \times 10^{5}$, or $20 \times 10^{5}$ cells/well and cultured for 14 days in $\alpha$-MEM containing $10 \% \mathrm{FCS}$, penicillin $(100 \mathrm{U} / \mathrm{ml})$, streptomycin (100 $\mu \mathrm{g} / \mathrm{ml}), 10 \mathrm{mM} \beta$-glycerophosphate, and ascorbic acid phosphate salt $(50 \mu \mathrm{g} / \mathrm{ml})$ for the assay of clonogenic osteoblast precursors. The wells containing TRAP-positive multinucleated cells (MNCs) ( $\geq 3$ nuclei/cell) or ALP-positive cells were counted as osteoclast- or osteoblast-positive after the TRAP or ALP staining using the leukocyte acid phosphatase kit (387A; Sigma) and ALP kit (86R; Sigma), respectively.

In vitro colony formation assay. The numbers of colony-forming unit-macrophages (CFU-M) in bone marrow were determined as described (21). Briefly, bone marrow cells $\left(1 \times 10^{4}\right)$ were cultured in $1 \mathrm{ml}$ of MEM containing $1.2 \%$ methylcellulose, 
Table II. Effects of ethanol on clinical characteristics.

Control Ethanol-fed

\begin{tabular}{lcc}
\hline Body weight $(\mathrm{g})$ & & \\
Start & $164.1 \pm 1.4$ & $162.5 \pm 0.6$ \\
Final & $196.0 \pm 2.1$ & $200.3 \pm 3.6$ \\
Food intake $(\mathrm{g} /$ day $)$ & $46.4 \pm 0.5$ & $46.2 \pm 0.7$ \\
Bone length $(\mathrm{mm})$ & & \\
Femur & $31.3 \pm 0.5$ & $31.2 \pm 0.4$ \\
Tibia & $35.3 \pm 0.1$ & $35.0 \pm 0.4$ \\
Bone weight $(\mathrm{g})$ & & \\
Femur & $0.553 \pm 0.009$ & $0.524 \pm 0.004^{\mathrm{a}}$ \\
Tibia & $0.414 \pm 0.004$ & $0.396 \pm 0.007^{\mathrm{a}}$ \\
Proximal tibia & $0.183 \pm 0.003$ & $0.174 \pm 0.003^{\mathrm{a}}$ \\
Serum & & \\
TBARS $(\mathrm{nmol} / \mathrm{ml})$ & $1.81 \pm 0.21$ & $3.84 \pm 0.41^{\mathrm{a}}$ \\
GSH $(\mu \mathrm{mol} / \mathrm{l})$ & $41.42 \pm 2.19$ & $35.44 \pm 1.04^{\mathrm{a}}$ \\
\hline
\end{tabular}

Values are means \pm SEM for 8 rats. ${ }^{a}$ Significantly different from the control value $(\mathrm{P}<0.05)$.

$30 \% \mathrm{FCS}, 1 \% \mathrm{BSA}$ and M-CSF (10 $\mathrm{ng} / \mathrm{ml})$ for 7 days, and the colonies $(>25 \mu \mathrm{m}$ in diameter) were counted.

Quantitative real-time $R T$-PCR. Total-RNA was prepared from the proximal tibia after the washing out of bone marrow cells and homogenizing in the presence of $0.1 \mathrm{M}$ EDTA or from cell lysate using a commercial kit (Sepasol-RNA I Super G; Nacalai Tesque, Inc., Kyoto, Japan). The total-RNA was reverse-transcribed with a first-strand cDNA synthesis kit (Toyobo, Co., Ltd., Tokyo, Japan). Real-time PCR was performed using the cDNA or total-RNA for the negative control, with Thunderbird ${ }^{\mathrm{TM}}$ SYBR qPCR Mix (Toyobo, Co., Inc.) and specific primers (Table I) as described (18). Levels of gene expression were determined relative to an internal standard (actin) and expressed relative to the control values.

Western blot analysis. Bone extracts of the proximal tibia (17) or cell lysates were used for the western blot analysis. The protein concentrations were measured using the BCA protein assay kit (Pierce of Thermo Fisher Scientific Inc., Rockford, IL). Equal amounts of protein were separated by sodium dodecyl sulfate-polyacrylamide gel electrophoresis and transferred to membranes. Western blotting and reprobing were performed and the chemiluminescent signals were quantified by a densitometer as reported (21). Antibodies recognizing actin (H-300), RANK (H-300), RANKL (FL-317), osteoprotegerin (OPG) (H-240), PU.1 (H-135), MITF (H-50) and p-ERK (E-4) were purchased from Santa Cruz Biotechnology, Inc. (Santa Cruz, CA). The anti-ERK antibody was obtained from Cell Signaling Technology, Inc. (Hitchin, UK).

Osteoclastic differentiation of the bone marrow-derived macrophage/monocyte precursor cells (BMMs). Bone marrow cells from the femur and tibia were cultured for $16-24 \mathrm{~h}$ in the
Table III. Effects of ethanol on bone biochemical markers.

\section{Control Ethanol-fed}

$\begin{array}{lcc}\text { Proximal tibia } & & \\ \text { ALP activity }(\mathrm{U} / \mathrm{g}) & 21.12 \pm 0.59 & 21.46 \pm 1.66 \\ \text { TRAP activity }(\mathrm{U} / \mathrm{g}) & 0.838 \pm 0.037 & 1.266 \pm 0.090^{\mathrm{a}} \\ \text { Cathepsin K activity }(\mathrm{U} / \mathrm{g}) & 3155.7 \pm 150.3 & 3868.8 \pm 203.6^{\mathrm{a}} \\ \mathrm{Ca}(\mathrm{mg} / \mathrm{g}) & 88.16 \pm 2.56 & 72.73 \pm 2.70^{\mathrm{a}} \\ \mathrm{Hyp}(\mu \mathrm{mol} / \mathrm{g}) & 104.1 \pm 2.20 & 93.2 \pm 1.50^{\mathrm{a}} \\ \text { Serum osteocalcin }(\mathrm{ng} / \mathrm{ml}) & 79.90 \pm 2.74 & 78.60 \pm 0.84\end{array}$

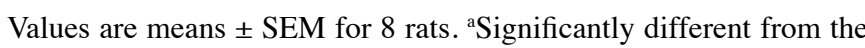
control value $(\mathrm{P}<0.05)$.

culture medium and cells at the interface after Ficoll-Paque gradient centrifugation of nonadherent cells were used as BMMs (10). BMMs $\left(1 \times 10^{4}\right.$ cells/well of a 96 -well plate or $1.5 \times 10^{5} \mathrm{cell} / \mathrm{s} / 35-\mathrm{mm}$ plate) (11) were cultured in the culture medium containing M-CSF $(20 \mathrm{ng} / \mathrm{ml})$ and RANKL $(10 \mathrm{ng} / \mathrm{ml})$ with or without ethanol. Cultures were maintained with a change of medium every 3 days. After 5 days, cells were used for TRAP staining and the assessment of cell viability with a leukocyte acid phosphatase kit (387A; Sigma) and WST-8 (Cell Counting kit-8; Dojindo Laboratories, Kumamoto, Japan), respectively, as described previously (10).

Determination of ERK activation, levels of RANK, PU.1 and $M I T F$, and production of intracellular reactive oxygen species during osteoclastic differentiation of BMMs. BMMs were precultured in the absence of M-CSF with or without ethanol or DPI $(50 \mu \mathrm{M})$ for $30 \mathrm{~min}$ and stimulated with M-CSF (20 ng/ $\mathrm{ml})$. Cells were harvested after stimulation for $5 \mathrm{~min}$ or $24 \mathrm{~h}$ and levels of phosphorylated ERK or RANK, PU.1 and MITF proteins were determined, respectively. To determine the generation of reactive oxygen species, cells were incubated for 10 min with 2', 7'-dichlorofluorescein diacetate (DCFH-DA) $(10 \mu \mathrm{M})$ after stimulation for $5 \mathrm{~min}$, and the fluorescence of DCF was detected by fluorescence microscopy as described previously (11).

Statistical analysis. All statistical analyses were performed by Welch's method using the Microsoft Excel data analysis program. The differences were considered significant at $\mathrm{P}<0.05$. All data are expressed as the mean \pm SEM.

\section{Results}

Effects of ethanol on clinical characteristics and bone biochemical markers. The ethanol-fed rats consumed an average of $2.3 \pm 0.03 \mathrm{~g}$ (mean $\pm \mathrm{SEM}$ ) of ethanol/day. Plasma ethanol concentrations were $111.9 \pm 10.6 \mathrm{mg} / \mathrm{dl}$, ranging from 80 to $142 \mathrm{mg} / \mathrm{dl}$. Body weight, food intake and bone length were similar to those in the control group, but the weights of the femur and tibia were significantly lower (Table II). The serum levels of TBARS and GSH were higher and lower than the control values, respectively. 
Table IV. Bone histomorphometry.

Control

$36.69 \pm 1.49$

$24.26 \pm 1.07$

$33.16 \pm 1.35$

$9.78 \pm 0.75$

$55.92 \pm 0.92$

$7.09 \pm 0.51$
Ethanol-fed

$28.13 \pm 1.35^{\mathrm{a}}$

$21.70 \pm 0.55^{\mathrm{a}}$

$25.85 \pm 0.63^{\mathrm{a}}$

$10.85 \pm 0.28$

$53.23 \pm 1.19$

$13.29 \pm 0.55^{\mathrm{a}}$

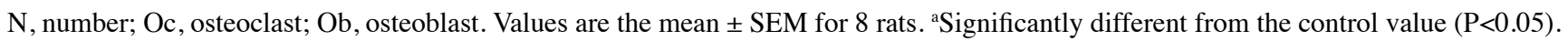

Table V. Frequency analysis of osteoclast and osteoblast precursors and CFU-M.

\begin{tabular}{lcc}
\hline & Control & Ethanol-fed \\
\hline Osteoclast precursor (cells/100 BMs) & $0.175 \pm 0.017$ & $0.480 \pm 0.028^{\mathrm{a}}$ \\
CFU-M (cells/104 BMs) & $68.0 \pm 5.9$ & $71.3 \pm 6.8$ \\
Osteoblast precursor (cells/10 $\mathrm{BMs})$ & $0.167 \pm 0.016$ & $0.183 \pm 0.029$ \\
\hline
\end{tabular}

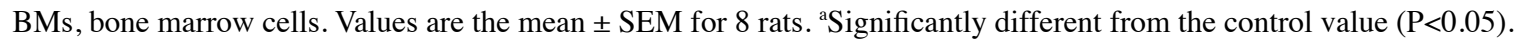

A

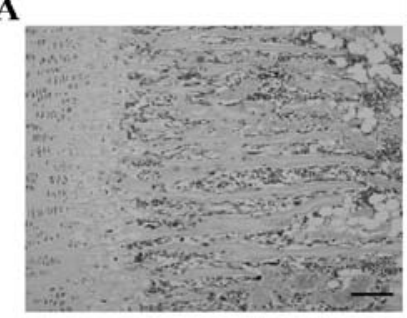

Control
B

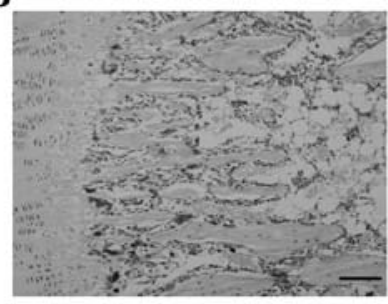

Ethanol-fed
Figure 1. TRAP staining of the proximal tibia in (A) control and (B) ethanolfed rats. Paraformaldehyde-fixed, decalcified and paraffin-embedded tibia obtained from (A) control and (B) the ethanol-fed rats, was processed for TRAP staining. TRAP-positive cells appeared red. Hematoxylin counterstaining. Magnification x200. Bar, $100 \mu \mathrm{m}$. The results presented here are typical of 4 rats of each group.
The ALP activity in the proximal tibia and serum osteocalcin levels were similar to those in the control group (Table III). The activities of TRAP and cathepsin K increased significantly to 1.5- and 1.2-fold the control level, respectively. The levels of hydroxyproline and $\mathrm{Ca}$ decreased to about $90 \%$ and $80 \%$ of the control value, respectively.

Histological analysis. The results of histochemical staining of the tibia for TRAP, a marker of osteoclasts, are shown in Fig. 1. Morphometric measurements showed that the number of osteoclasts in the ethanol-fed rats increased to 1.9-fold the control value (Table IV). The number of osteoblasts was unchanged. Trabecular bone volume, surface and thickness were decreased, but trabecular numbers were not significantly affected.

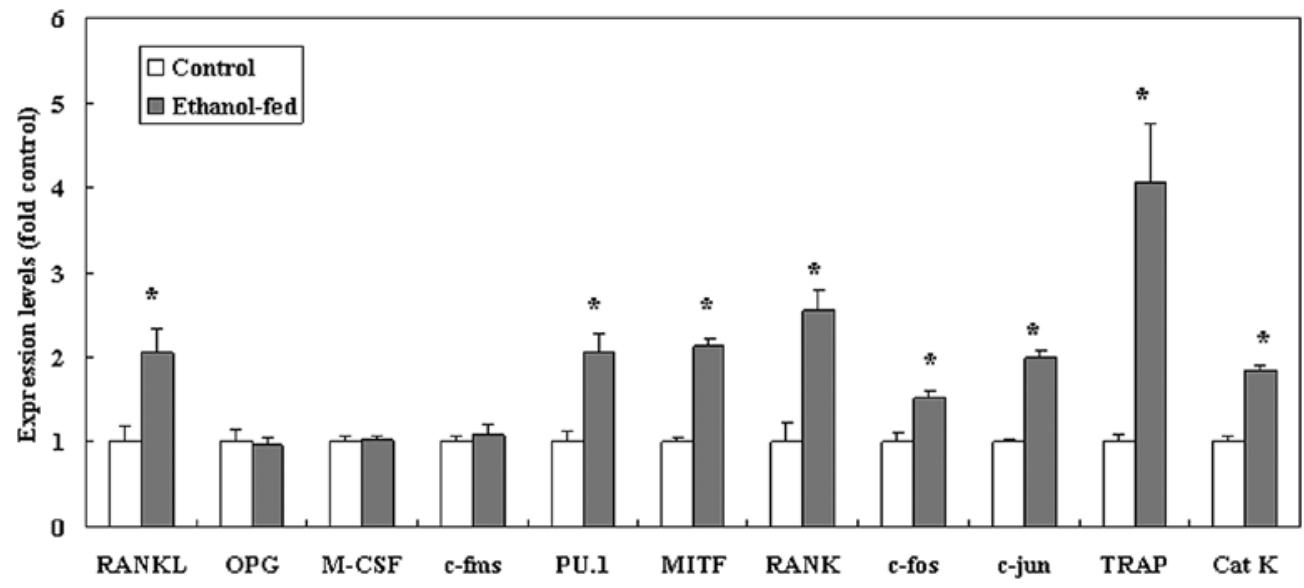

Figure 2. Expression of genes involved in osteoclastic differentiation in the proximal tibia. Total-RNA was extracted from the proximal tibia of control and ethanol-fed rats and the mRNA levels of RANKL, OPG, M-CSF, c-fms, PU.1, MITF, RANK, c-fos, c-jun, TRAP and cathepsin K (Cat K) were assessed by quantitative real-time PCR as described in Materials and methods. Values are the mean \pm SEM for 8 rats. Levels are expressed relative to the control value (fold-increase). * Significantly different from the control value $(\mathrm{P}<0.05)$. 
$\mathbf{A}$

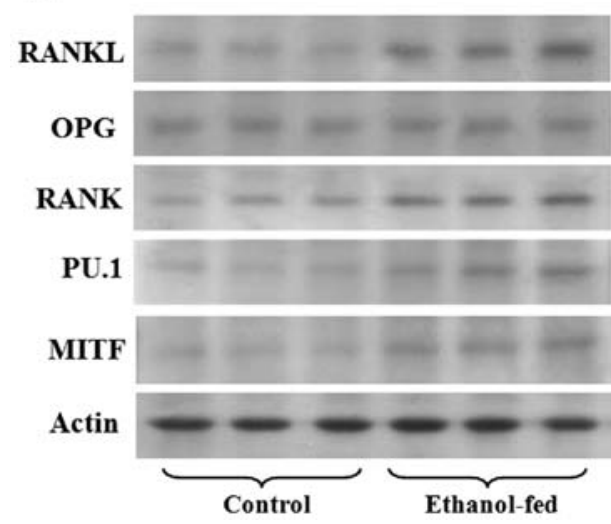

B

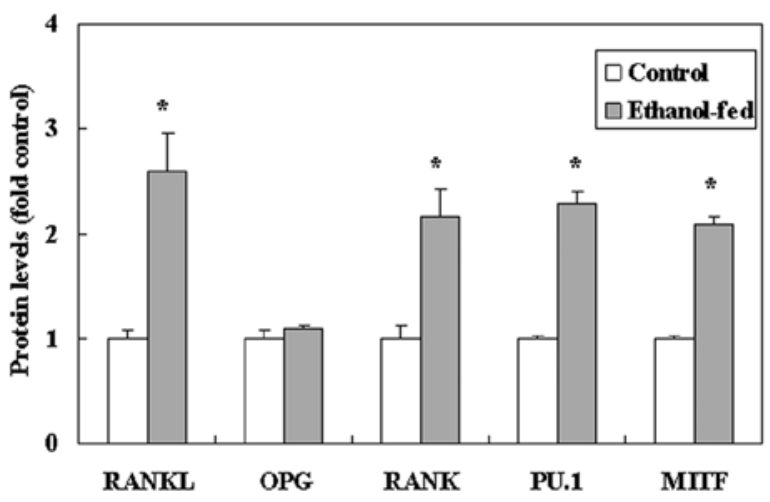

C

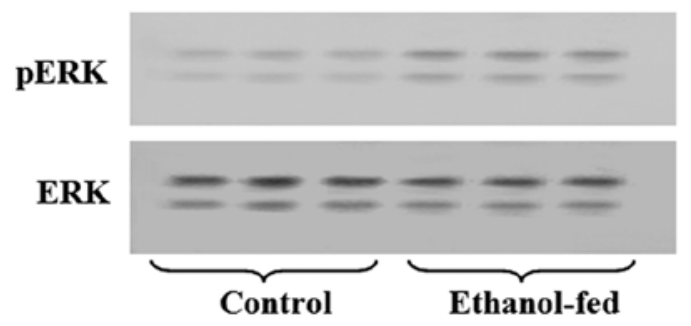

D

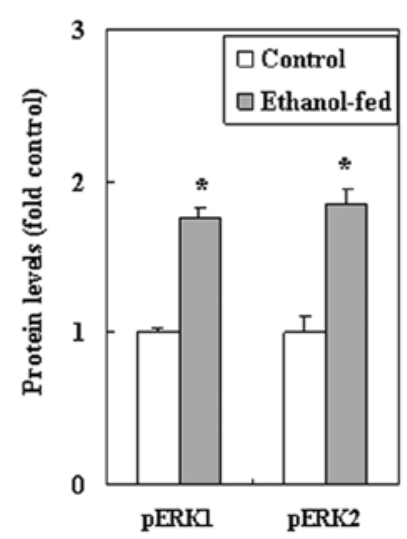

Figure 3. Protein levels of RANKL, OPG, RANK, PU.1, MITF and phosphorylated ERK in the proximal tibia. Bone extracts from the proximal tibia of control and ethanol-fed rats were resolved by SDS-PAGE. After transfer, the blot was probed or reprobed with antibody and detected by ECL as described in Materials and methods. Western blot analyses; (A) RANKL, OPG, RANK, PU.1 and MITF, (C) phosphorylated ERK. The protein levels were quantified by densitometry and represented graphically $(B, D)$. Data represent the mean \pm SEM for 8 rats. "Significantly different from the control value $(\mathrm{P}<0.05)$.
Effects of ethanol on the population of osteoclast and osteoblast precursors and CFU-M in bone marrow. Based on the frequency analysis, the population of osteoclast precursor cells in bone marrow (frequency) in the ethanol-fed rats increased to 2.7-fold the control level, although no significant difference was observed in the population of osteoblast precursors (Table V). The numbers of CFU-M in bone marrow were similar among the 2 groups.

Expression of genes involved in osteoclastic differentiation in the proximal tibia. The gene expression levels of the osteoclastogenesis-related factors, RANKL, OPG, M-CSF, c-fms, PU.1, MITF, RANK, c-fos, and c-jun and osteoclast-specific proteins, TRAP and cathepsin K, relative to the internal control, actin, are shown in Fig. 2. The mRNA level of RANKL was about 2-fold the control value (Fig. 2). The expression of OPG, $\mathrm{M}$-CSF and c-fms were not different from the control values. However, levels of RANK, PU.1, MITF, c-fos and c-jun in the ethanol-fed rats were 2.5-, 2-, 2-, 1.5- and 2-fold the control values, respectively. The expression of TRAP and cathepsin K mRNA also increased to 4- and 1.8-fold the control values, respectively.

Protein Levels of RANKL, OPG, RANK, PU.1, MITF and phosphorylated ERK in the proximal tibia. The results of the western blot analysis are shown in Fig. 3. The protein levels of RANKL and RANK in the ethanol-fed group increased to 2.5- and 2-fold the control values, respectively, while the OPG level was similar to the control value (Fig. $3 \mathrm{~A}$ and B). The PU.1 and MITF protein levels also increased to about 2-fold the control value. The level of phosphorylated ERK1/2 in the EtOH rats was 1.8-fold the control value (Fig. $3 \mathrm{C}$ and D).

Effects of ethanol on osteoclastic differentiation and the protein levels of RANK, PU.1, MITF and phosphorylated ERK in BMMs. To clarify the direct effects of ethanol on osteoclast precursor cells, an experiment using BMMs was performed. The addition of ethanol stimulated the osteoclastic differentiation induced by M-CSF and RANKL in BMMs as shown in Fig. 4A. Ethanol at 25 and $50 \mathrm{mM}$ increased the number of TRAP-positive cells to 1.4- and 1.6-fold the control level, respectively (Fig. 4B). Cell viability was not affected by the presence of ethanol (Fig. 4C).

The levels of RANK protein increased to 2.5 -fold the control level in the presence of 25 or $50 \mathrm{mM}$ ethanol (Fig. 4D). PU.1 levels increased to 1.6-, 3- and 3-fold on exposure to 10,25 and $50 \mathrm{mM}$ ethanol, respectively. The levels of MITF increased to 2.5- and 3-fold the control in the presence of 25 and $50 \mathrm{mM}$ ethanol, respectively.

As M-CSF-induced RANK expression was mediated through the activation of ERK (10), the levels of phosphorylated ERK were examined in the presence of ethanol. The phosphorylation of ERK 1/2 increased at $5 \mathrm{~min}$ after the addition of M-CSF (Fig. 4E). The presence of 10, 25 and $50 \mathrm{mM}$ of ethanol increased the phosphorylation of ERK1 1.5-, 3- and 3.6-fold, and ERK2 levels 1.6-, 2.6- and 3-fold, respectively, relative to the control value, respectively.

Effects of ethanol on the generation of ROS during the osteoclastogenesis of BMMs. The intracellular production 
A

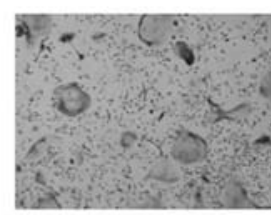

Control

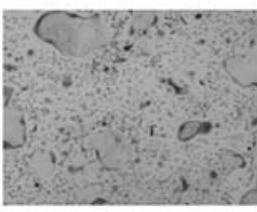

Ethanol (10 mM)

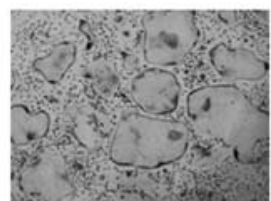

Ethanol (25 mM)

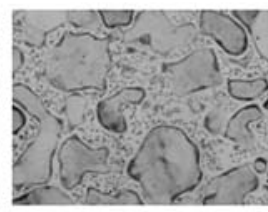

Ethanol (50 mM)
B

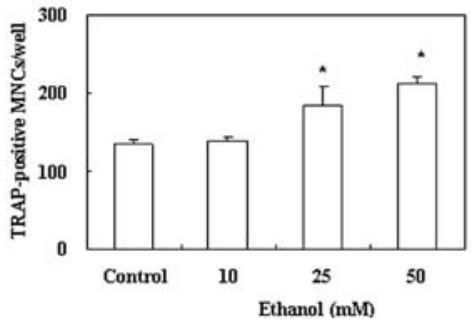

D

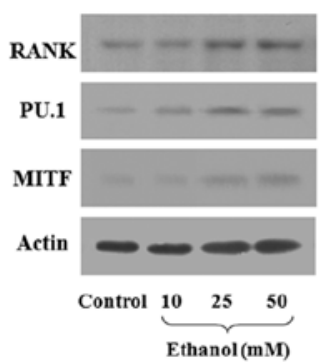

C

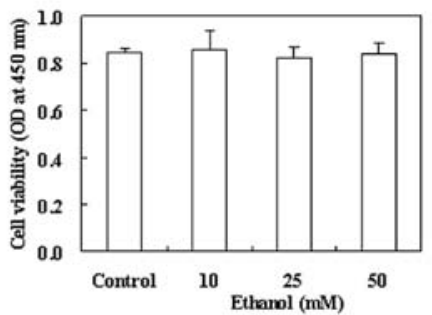

$\mathbf{E}$

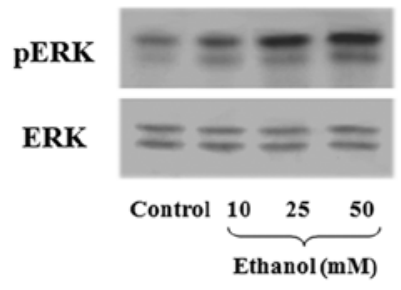

Figure 4. Effects of ethanol on osteoclastic differentiation of BMMs and the protein levels of RANK, PU.1, MITF and phosphorylated ERK. (A-C) BMMs were cultured with M-CSF and RANKL in the absence or presence of ethanol (10,25 or $50 \mathrm{mM}$ ). After the culture of BMMs for 5 days, the cells were used for TRAP staining and the assessment of cell viability as described in Materials and methods. The photographs (x100 magnification) are representative of 6 experiments. (D) After the culture of BMMs for $24 \mathrm{~h}$, the cells were harvested and used for western blotting for RANK, PU1 or MITF. (E) After the culture of BMMs for $5 \mathrm{~min}$, the cells were harvested and used for western blotting for ERK1/2 or phosphorylated ERK. The protein levels are shown relative to the control value. Values are the mean \pm SEM for 6 experiments . "Significantly different from the control value $(\mathrm{P}<0.05)$.

$\mathbf{A}$

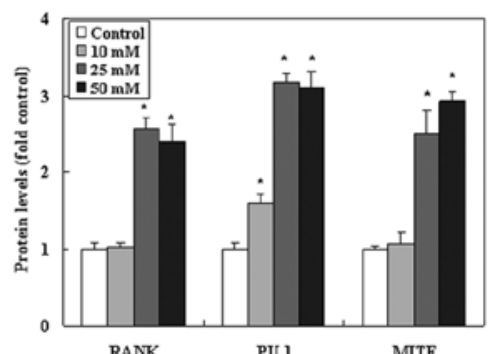

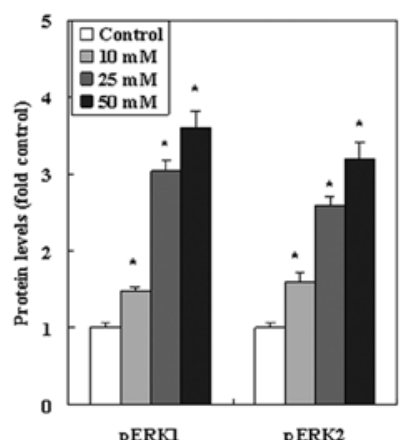

PERK
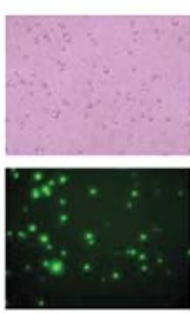

$+$

$+$
M-CSF

DPI

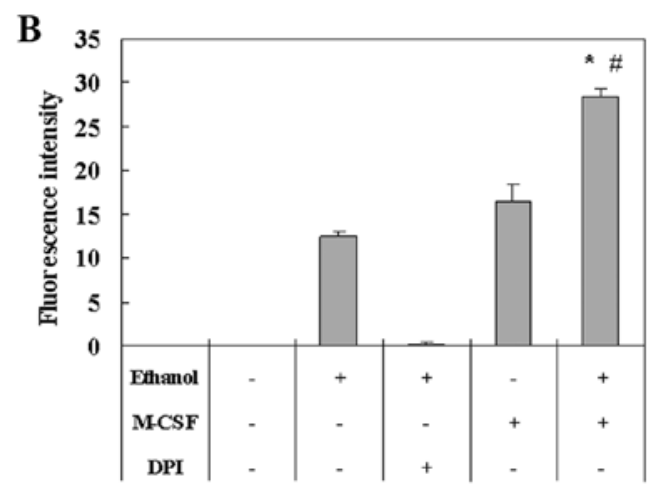

Figure 5. Effects of ethanol on the generation of reactive oxygen species during the osteoclastogenesis of BMMs. BMMs were pre-cultured with or without ethanol $(25 \mathrm{mM})$ or DPI $(50 \mu \mathrm{M})$ for $30 \mathrm{~min}$. After stimulation with M-CSF $(20 \mathrm{ng} / \mathrm{ml})$ for $5 \mathrm{~min}$ or no stimulation, BMMs were incubated with DCFH-DA for $10 \mathrm{~min}$. DCF fluorescence was detected with fluorescence microscopy. (A) Typical pictures (x400 magnification) and (B) quantitative calculation data are shown. Results are representative of 8 independent sets of similar experiments and expressed as the mean \pm SEM. ${ }^{*}$ Significantly different from the $(+\mathrm{M}-\mathrm{CSF})$ value $(\mathrm{P}<0.05)$. "Significantly different from the $(+\mathrm{Ethanol})$ value $(\mathrm{P}<0.05)$. 
of ROS was measured with DCFH. Exposure to $25 \mathrm{mM}$ of ethanol resulted in an increase in the intensity of fluorescence (Fig. 5A). The presence of DPI, a Nox inhibitor, suppressed the increase in fluorescence induced by ethanol. The stimulation of BMMs with M-CSF increased the intensity of DCF fluorescence. Exposure to ethanol combined with the stimulation by $\mathrm{M}-\mathrm{CSF}$ induced an additive increase in fluorescence intensity (Fig. 5).

\section{Discussion}

This study clearly demonstrated that ethanol increased the activity and number of osteoclasts. No significant change in the activity or number of osteoblasts was observed after 3 weeks of ingesting ethanol. This is consistent with a report that osteoblast function in cancellous bone of rapidly growing rats (at 6 weeks or younger) appeared to be unaffected during 2-8 weeks of exposure to ethanol (22). The histological analysis showed that the increase in osteoclastic activity was associated with the number of osteoclasts, although the osteoblast number was unchanged. The frequency assay also showed an increase in osteoclast precursors, but no significant change in osteoblast precursors, in bone marrow of the ethanol-fed rats. These results suggested that ethanol did not affect the numbers of osteoblasts/precursors but increased the numbers of osteoclasts and their precursors. However, a significant difference in CFU-M was not observed between the two groups. In bone marrow, the phenotype of progenitors for osteoclasts was comparable with that of CFU-M (23-25). These results suggested that ethanol had no effect on the population of precursors which responded to M-CSF, but reduced that of the precursors which differentiated into osteoclasts in response to RANKL.

The precursors of osteoclasts are derived from the hematopoietic stem cells in bone marrow. The differentiation into osteoclasts, however, occurs on the bone surface in vivo $(2,24)$. Therefore, the gene expression of osteoclastogenesisrelated factors was examined in the bone. Consistent with the frequency analysis, the mRNA levels of M-CSF and c-fms, the receptor of M-CSF, in the ethanol-fed rats were similar to the control values. However, the mRNA and protein levels of RANKL were significantly increased, although the OPG level was unchanged. RANKL plays a critical role in the differentiation of osteoclast precursors into osteoclasts. Osteoclastic differentiation is principally stimulated by an increase in the biological availability of RANKL, assessed by the ratio of RANKL to its decoy receptor, OPG (26-28). The ratio of RANKL to OPG in the ethanol-fed rats was significantly increased. Consistent with previous reports $(6,14)$, ethanol stimulated osteoclastogenesis through an increase in RANKL expression in osteoblasts. However, in the present study, the mRNA and protein levels of RANK, the receptor of RANKL, also increased. These findings suggested that the increase in osteoclastogenesis caused by ethanol was due to an increase in the expression of RANKL in osteoblasts/stromal cells and also RANK in osteoclast precursors. In this study, for the first time, an increase in the mRNA and protein levels of RANK as well as RANKL due to ethanol was shown. Further, the increase in RANK expression was associated with increases in the mRNA and protein levels of PU.1 and MITF. The gene expression of RANK was shown to be regulated by the tran- scription factors PU.1 and MITF (29). These results suggested that ethanol increased the expression of RANK through the increase in PU.1 and MITF.

To investigate whether ethanol acted directly on the osteoclast progenitor cells, experiments were performed in vitro using BMMs. Ethanol stimulated the osteoclastic differentiation of BMMs and simultaneously increased the expression of RANK, PU.1 and MITF. These results indicated that ethanol acted directly on osteoclast precursors and stimulated osteoclastic differentiation through the increased expression of RANK. Further, the increased expression of RANK was associated with the activation of ERK in agreement with our previous report (10). Indeed, the activation of ERK was also observed here in the proximal tibia of the ethanol-fed rats, in this study, although it is not clear where it occurred, in osteoclasts, osteoblasts or their precursors, in vivo. It was reported that ethanol activated ERK in osteoblasts (14). These results suggested that the activation of ERK occurred in ostoclasts/ precursors as well as osteoblasts of the ethanol-fed rats.

ROS was reported to play an important role in the activation of ERK in osteoblasts $(14,30)$. In the present study, exposure to ethanol induced the production of ROS in BMMs. The Nox inhibitor DPI inhibited ROS production, indicating the NADPH-dependent generation of ROS in BMMs. Nox has been implicated in the activation of several signaling cascades, including ERK-signaling pathways (31). In fact, ethanolinduced ROS generation via NADPH was also reported in osteoblasts (14). These results suggested that ethanol generated ROS via a NADPH-dependent pathway, activated ERK, and induced the expression of RANK in osteoclast precursors.

Bone formation has long been believed to be the primary target of alcohol's deleterious effects on bone metabolism (1). However, early on, the ethanol acted directly on osteoclast precursors. This study have provided evidence that ethanol stimulates osteoclastogenesis through the increased expression of RANK mediated by the production of ROS and activation of ERK in osteoclast precursor cells in addition to an increase in RANKL in osteoblast stromal cells.

\section{References}

1. Turner RT: Skeletal response to alcohol. Alcohol Clin Exp Res 24: 1693-1701, 2000

2. Manolagas SC: Birth and death of bone cells: basic regulatory mechanisms and implications for the pathogenesis and treatment of osteoporosis. Endocr Rev 21: 115-137, 2000.

3. Rodan GA and Martin TJ: Therapeutic approaches to bone diseases. Science 289: 1508-1514, 2000.

4. Chakkalakal DA: Alcohol-induced bone loss and deficient bone repair. Alcohol Clin Exp Res 29: 2077-2090, 2005.

5. Callaci JJ, Himes R, Lauing K, Wezeman FH and Brownson K: Binge alcohol-induced bone damage is accompanied by differential expression of bone remodeling-related genes in rat vertebral bone. Calcif Tissue Int 84: 474-484, 2009.

6. Dai J, Lin D, Zhang J, et al: Chronic alcohol ingestion induces osteoclastogenesis and bone loss through IL- 6 in mice. J Clin Invest 106: 887-895, 2000.

7. Udagawa N, Takahashi N, Akatsu T, et al: Origin of osteoclasts: mature monocytes and macrophages are capable of differentiating into osteoclasts under a suitable microenvironment prepared by bone marrow-derived stromal cells. Proc Natl Acad Sci USA 87: 7260-7264, 1990.

8. Harada S and Rodan GA: Control of osteoblast function and regulation of bone mass. Nature 423: 349-355, 2003.

9. Asagiri $\mathrm{M}$ and Takayanagi $\mathrm{H}$ : The molecular understanding of osteoclast differentiation. Bone 40: 251-264, 2007. 
10. Hie $\mathrm{M}$ and Tsukamoto I: Vitamin C-deficiency stimulates osteoclastogenesis with an increase in RANK expression. J Nutr Biochem 22: 164-171, 2011.

11. Hie $\mathrm{M}$ and Tsukamoto I: Administration of zinc inhibits osteoclastogenesis through the suppression of RANK expression in bone. Eur J Pharmacol 668: 140-146, 2011.

12. Novitskiy G, Traore K, Wang L, Trush MA and Mezey E: Effects of ethanol and acetaldehyde on reactive oxygen species production in rat hepatic stellate cells. Alcohol Clin Exp Res 30: $1429-1435,2006$.

13. Polikandriotis JA, Rupnow HL, Elms SC, et al: Chronic ethanol ingestion increases superoxide production and NADPH oxidase expression in the lung. Am J Respir Cell Mol Biol 34: 314-319, 2006.

14. Chen JR, Shankar K, Nagarajan S, Badger TM and Ronis MJ: Protective effects of estradiol on ethanol-induced bone loss involve inhibition of reactive oxygen species generation in osteoblasts and downstream activation of the extracellular signalregulated kinase/signal transducer and activator of transcription $3 /$ receptor activator of nuclear factor-kappaB ligand signaling cascade. J Pharmacol Exp Ther 324: 50-59, 2008.

15. Ohkawa $\mathrm{H}$, Ohishi $\mathrm{N}$ and Yagi K: Assay for lipid peroxides in animal tissues by thiobarbituric acid reaction. Anal Biochem 95: 351-358, 1979

16. Ellman GL: Tissue sulfhydryl groups. Arch Biochem Biophys 82: 70-77, 1959.

17. Goto A and Tsukamoto I: Increase in tartrate-resistant acid phosphatase of bone at the early stage of ascorbic acid deficiency in the ascorbate-requiring Osteogenic Disorder Shionogi (ODS) rat Calcif Tissue Int 73: 180-185, 2003.

18. Hie M, Shimono M, Fujii K and Tsukamoto I: Increased cathepsin $\mathrm{K}$ and tartrate-resistant acid phosphatase expression in bone of streptozotocin-induced diabetic rats. Bone 41: 10451050, 2007.

19. Parfitt AM, Drezner MK, Glorieux FH, et al: Bone histomorphometry: standardization of nomenclature, symbols, and units. Report of the ASBMR Histomorphometry Nomenclature Committee. J Bone Miner Res 2: 595-610, 1987.

20. Sato T, Shibata T, Ikeda K and Watanabe K: Generation of boneresorbing osteoclasts from B220+ cells: its role in accelerate osteoclastogenesis due to estrogen deficiency. J Bone Miner Res 16: 2215-2221, 2001.
21. Hie M, Yamazaki M and Tsukamoto I: Curcumin suppresses increased bone resorption by inhibiting osteoclastogenesis in rats with streptozotocin-induced diabetes. Eur J Pharmacol 621: 1-9, 2009.

22. Sampson HW, Chaffin C, Lange J and DeFee B II: Alcohol consumption by young actively growing rats: a histomorphometric study of cancellous bone. Alcohol Clin Exp Res 21: 352-359, 1997.

23. Sudo T, Nishikawa S, Ogawa M, et al: Functional hierarchy of $\mathrm{c}-\mathrm{kit}$ and c-fms in intramarrow production of CFU-M. Oncogene 11: 2469-2476, 1995.

24. Hayashi S, Miyamoto A, Yamane T, et al: Osteoclast precursors in bone marrow and peritoneal cavity. J Cell Physiol 170: 241-247, 1997.

25. Arai F, Miyamoto T, Ohneda O, et al: Commitment and differentiation of osteoclast precursor cells by the sequential expression of c-Fms and receptor activator of nuclear factor kappaB (RANK) receptors. J Exp Med 190: 1741-1754, 1999.

26. Suda T, Takahashi N, Udagawa N, Jimi E, Gillespie MT and Martin TJ: Modulation of osteoclast differentiation and function by the new members of the tumor necrosis factor receptor and ligand families. Endocr Rev 20: 345-357, 1999.

27. Riggs BL: The mechanisms of estrogen regulation of bone resorption. J Clin Invest 106: 1203-1204, 2000.

28. Hofbauer LC, Khosla S, Dunstan CR, Lacey DL, Boyle WJ and Riggs BL: The roles of osteoprotegerin and osteoprotegerin ligand in the paracrine regulation of bone resorption. $J$ Bone Miner Res 15: 2-12, 2000.

29. Ishii J, Kitazawa R, Mori K, et al: Lipopolysaccharide suppresses RANK gene expression in macrophages by down-regulating PU.1 and MITF. J Cell Biochem 105: 896-904, 2008.

30. Bai XC, Lu D, Liu AL, et al: Reactive oxygen species stimulates receptor activator of NF-kappaB ligand expression in osteoblast. J Biol Chem 280: 17497-17506, 2005.

31. Jackson SH, Devadas S, Kwon J, Pinto LA and Williams MS: $T$ cells express a phagocyte-type NADPH oxidase that is activated after $\mathrm{T}$ cell receptor stimulation. Nat Immunol 5: 818-827, 2004 